\title{
Influencia de la presencia de fases secundarias en las propiedades piezoeléctricas de materiales cerámicos basados en PZT
}

\author{
T. JARDIEL, J.F. FERNÁNDEZ, A.C. CABALLERO, M. VILLEGAS \\ Departamento de Electrocerámica. Instituto de Cerámica y Vidrio, CSIC, 28500 Arganda del Rey, Madrid
}

\begin{abstract}
Se ha realizado un estudio comparativo de la microestructura, las propiedades eléctricas y piezoeléctricas de un material cerámico PZT tipo-5A sin modificar y modificado con un exceso de $\mathrm{Nb}_{2} \mathrm{O}_{5}$. Mediante Microscopía Electrónica de Barrido se observa que el PZT modificado presenta una estructura más heterogénea. Se han caracterizado las propiedades dieléctricas y piezoeléctricas de los materiales con y sin adición de $\mathrm{Nb}_{2} \mathrm{O}_{5}$ y se ha determinado la influencia de la presencia de fases secundarias sobre la variación de la Temperatura de Curie y el desplazamiento de la composición del PZT debido a la extracción de $\mathrm{PbO}$ desde la matriz de PZT.
\end{abstract}

Palabras clave: Titanato Circonato de Plomo, Microestructura, Temperatura de Curie.

Influence of the presence of secondary phases on the piezoelectric properties of PZT-based ceramics

A comparative study of the microstructure, the dielectric, and the piezoelectric properties of PZT-5A type ceramics unmodified and modified with an excess of $\mathrm{Nb}_{2} \mathrm{O}_{5}$ has been carried out. By SEM, the microstructure of the modified ceramics appears more heterogeneous than that corresponding to the unmodified one. The piezoelectric and dielectric properties of both materials, with and without $\mathrm{Nb}_{2} \mathrm{O}_{5}$ addition, have been measured. The influence of the secondary phases on the variation of the Curie temperature has been determined, as well as the displacement of the PZT composition due to extraction of $\mathrm{PbO}$ from the PZT matrix.

Key words: Lead Titanate Zirconate, Microstructure, Curie Temperature.

\section{INTRODUCCIÓN}

Los materiales cerámicos basados en titanato circonato de plomo, $\mathrm{Pb}\left(\mathrm{Zr}_{\mathrm{x}} \mathrm{Ti}_{1-\mathrm{x}}\right) \mathrm{O}_{3}(\mathrm{PZT})$, se utilizan como elementos piezoeléctricos en una amplia variedad de aplicaciones. En el rango de composición de la frontera morfotrópica de fases estos materiales presentan los coeficientes piezoeléctricos más elevados $(1,2)$. El aumento de las propiedades piezoeléctricas en las proximidades de la frontera morfotrópica de fases se debe a un aumento en la facilidad de reorientación de los dominios durante la polarización causado por la proximidad de las estructuras ferroeléctricas tetragonal y romboédrica, ya que es mayor el número de direcciones disponibles para la polarización, y por un máximo en la complianza mecánica.

La presencia de fases secundarias influye notablemente sobre sus propiedades dieléctricas y piezoeléctricas. Las cerámicas de PZT se usan casi siempre con adiciones de dopantes, modificadores u otros constituyentes químicos, a fin de mejorar y optimizar sus excelentes propiedades básicas. El $\mathrm{Nb}^{+5}$ clasifica dentro del grupo de los dopantes donadores. Éstos son iones cuya valencia es mayor que la del ión que reemplazan en la red. Los donores producen vacantes de plomo para mantener la electroneutralidad local, lo que facilita el movimiento de las paredes de dominio (3) y explica muchas de las propiedades de este grupo de aditivos. Sin embargo, la adición de $\mathrm{Nb}^{5+}$ provoca una disminución del tamaño de grano y de la densidad en el material, y conduce a la formación de
$\mathrm{ZrO}_{2}$ como fase secundaria (4-5).

El efecto de las pérdidas de $\mathrm{PbO}$, por sustituciones en la red o por evaporación a alta temperatura, también ha de tenerse en cuenta ya que influye en la composición y, por tanto, en las propiedades finales del material cerámico. La evaporación de $\mathrm{PbO}$ a alta temperatura provoca una reacción de descomposición (6) que hace que el material se aleje de la composición del límite morfotrópico de fase y por tanto que sus propiedades se degraden.

El objetivo de este trabajo ha sido estudiar cómo la incorporación de un exceso de $\mathrm{Nb}_{2} \mathrm{O}_{5}$ y la formación de fases secundarias en una matriz de $\mathrm{PZT}^{2}$ (53/47) dopado con $\mathrm{Nb}_{2} \mathrm{O}_{5}$ afecta a las propiedades finales de la piezocerámica. Para ello, el estudio se ha centrado en el efecto de la adición de un exceso de $\mathrm{Nb}_{2} \mathrm{O}_{5}$ y del procesamiento adicional que conlleva dicha modificación sobre la sinterización, propiedades y desplazamiento de la composición del PZT.

\section{PROCEDIMIENTO EXPERIMENTAL}

Ambos materiales fueron preparados a partir de un PZT tipo 5A de composición nominal $\mathrm{Pb}_{0.97} \mathrm{Sr}_{0.03}\left(\mathrm{Zr}_{0.53} \mathrm{Ti}_{0.455} \mathrm{Nb}_{0.015}\right) \mathrm{O}_{3}$ cuyo proceso de preparación se ha descrito con anterioridad (7) y con las especificaciones recogidas en la Tabla I. El mate- 
rial base se denominó A1. El material A2 se obtuvo añadiendo un exceso de $0.07 \%$ molar de $\mathrm{Nb}_{2} \mathrm{O}_{5}$ (Fluka) molido en atrición con microbolas, con un tamaño de partícula medio de $0.5 \mu \mathrm{m}$. Se empleó un óxido de niobio de tamaño medio de partícula pequeño para favorecer su incorporación a la matriz del PZT. La modificación del PZT-5A se llevó a cabo preparando una suspensión en medio etanólico con el material A1, añadiendo el exceso de $\mathrm{Nb}_{2} \mathrm{O}_{5}$ y realizando una mezcla mecánica mediante el uso de una turbina de alta velocidad de ciza1la. Posteriormente, la mezcla se secó en una estufa a $60^{\circ} \mathrm{C}$ y se tamizo a $100 \mu \mathrm{m}$.

Los polvos cerámicos A1 y A2 se conformaron en discos de $1.5 \mathrm{~cm}$ de diámetro y $0.3 \mathrm{~cm}$ de espesor mediante prensado uniaxial a $115 \mathrm{MPa}$. En primer lugar se realizó un primer tratamiento térmico a $600^{\circ} \mathrm{C}$ durante una hora para eliminar el aglomerante utilizado en el prensado. Los materiales en verde se caracterizaron mediante porosimetría de mercurio usando un porosímetro Autopore II 9220 de la casa Micromeritics.

La sinterización se llevó a cabo en un crisol sellado con $\mathrm{ZrO}_{2}$, sin emplear atmósfera rica en $\mathrm{PbO}$, a temperaturas entre $1050^{\circ}$ y $1200^{\circ} \mathrm{C}$ durante 4 horas. La densidad de los discos sinterizados se determinó por el método de Arquímedes, siendo el medio líquido el agua. Las pérdidas de peso se determinaron por diferencia de peso entre le material en verde después de eliminar el aglomerante y el material sinterizado. La microestructura se observó por Microscopía Electrónica de Barrido (MEB) sobre la superficie sin pulir ni atacar ("as-sintered") de las muestras sinterizadas usando un microscopio Carl Zeiss DSM 950. El tamaño de grano se determinó mediante el método de intercepción de línea (8) y las distintas fases presentes se analizaron mediante EDS. La presencia de fases secundarias se estudió también mediante Difracción de Rayos X (Siemens D5000) sobre la superficie de las muestras sinterizadas.

La caracterización dieléctrica y piezoeléctrica se realizó sobre los discos pulidos hasta alcanzar el plano-paralelismo y electrodados con pasta de plata. El electrodo se sinterizó a $750^{\circ} \mathrm{C}$ durante $15 \mathrm{~min}$. El proceso de polarización se realizó a un campo de $40 \mathrm{kV} / \mathrm{cm}$, a una temperatura de $120^{\circ} \mathrm{C}$ durante 30 minutos. La determinación de las propiedades dieléctricas y piezoeléctricas se realizó mediante un analizador vectorial de impedancias HP4192A. Las propiedades piezoeléctricas se evaluaron de acuerdo con las normas IEEE (9), empleando el método de resonancia-antiresonancia. La determinación del parámetro $\mathrm{d}_{33}$ se realizó en un equipo Berlincourt piezo-d meter.

\section{RESULTADOS Y DISCUSIÓN}

\subsection{Sinterización}

La figura 1.a muestra las curvas de densidad en función de la temperatura de sinterización para ambos materiales. La densificación sigue la misma tendencia en los dos casos, pero en el intervalo de temperaturas entre $1050^{\circ}$ y $1150^{\circ} \mathrm{C}$ el material con exceso de $\mathrm{Nb}_{2} \mathrm{O}_{5}$ alcanza densidades menores. Esto se puede atribuir a su diferente modo de procesado, ya el turbinado realizado sobre el material A2 produce la rotura de aglomerados haciendo que la distribución de tamaño de partícula sea más uniforme. Esto a su vez produce que el empaquetamiento sea peor que en el caso del material A1, donde la distribución más bimodal de tamaño de partícula favorece dicho empaquetamiento, $y$, así, que la densidad en verde del material A2 sea menor $\left(58,9 \pm 0.3 \% \mathrm{~d}_{\mathrm{t}}\right)$ que la del A1 $\left(61.6 \pm 0.3 \% \mathrm{~d}_{\mathrm{t}}\right)$.
TABLA I. EsPeCIFICACIONES DEL PZT TIPO 5A

\begin{tabular}{|cc|}
\hline Propiedad & Magnitud \\
\hline Temperatura de Curie & $360{ }^{\circ} \mathrm{C}$ \\
Coeficientes piezoeléctricos: & \\
$\mathrm{d}_{31}$ & $-(235 \pm 21) \mathrm{pC} / \mathrm{N}$ \\
$\mathrm{d}_{33}$ & $(374 \pm 7) \mathrm{pC} / \mathrm{N}$ \\
Permitividad dieléctrica a $25^{\circ} \mathrm{C}, \varepsilon_{\mathrm{r}}$ & $1792 \pm 61$ \\
P rdidas dieléctricas a $25^{\circ} \mathrm{C}, \mathrm{D}$ & $0.0133 \pm 0.0011$ \\
\hline
\end{tabular}
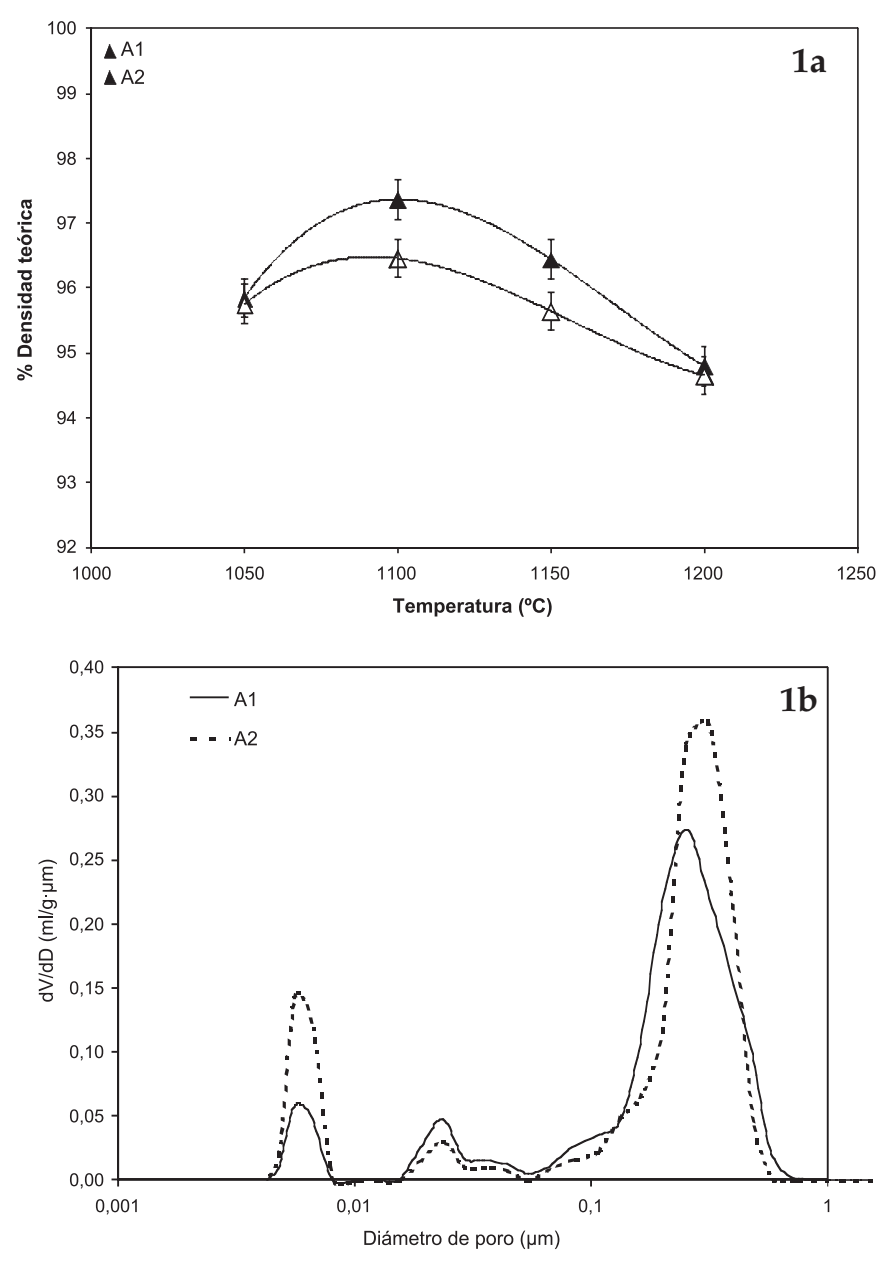

Figura 1. a) Variación de la densidad en función de la temperatura de sinterización de los materiales A1 y A2. b) Distribución de porosidad en discos prensados de los materiales A1 y A2 en verde.

Además, la distribución de porosidad de los materiales prensados en verde es también diferente (Figura 1.b). Como ya indicaba la densidad en verde, la porosidad es mayor en el material A2 y se produce un aumento de los poros interpartícula que son más difíciles de eliminar durante la sinterización. Además, la adición de $\mathrm{Nb}^{5+}$ dificulta la difusión provocando también una disminución de la densidad tras la sinterización (5). Estos hechos justifican que el comportamiento de densificación de ambos materiales sea comparable, pero que el material A2 alcance densidades menores. A alta temperatura $\left(1200^{\circ} \mathrm{C}\right)$, los valores de densidad de los materiales A1 y A2 disminuyen fuertemente debido al proceso de descomposición térmica que se produce por la volatilización del $\mathrm{PbO}$ (proceso de de-sinterización que comienza a observarse ya a $1150^{\circ} \mathrm{C}$ ) y que se relaciona con las pérdidas de peso mostradas 
en la Figura 1.c. Como puede observarse las pérdidas de peso en ambos materiales también siguen la misma tendencia. A bajas temperaturas de sinterización estas pérdidas se producen principalmente por la extracción de $\mathrm{PbO}$ provocada por la entrada de $\mathrm{Nb}_{2} \mathrm{O}_{5}$ en la matriz de PZT (generación de vacantes de $\mathrm{Pb}^{2+}$ ). Sin embargo, a altas temperaturas la descomposición del material es la causa principal de las pérdidas de peso. La diferencia de pérdida de peso teórica por la incorporación del exceso de $\mathrm{Nb}_{2} \mathrm{O}_{5}$ en el material A2 respecto del A1 es muy pequeña $(0.024 \%)$, sin embargo, la diferencia experimental es siempre mayor. Esto puede indicar que la presencia de un exceso de $\mathrm{Nb}_{2} \mathrm{O}_{5}$ favorece la extracción de $\mathrm{PbO}$ de la matriz (10). Además, la presencia de una mayor cantidad de porosidad en el material modificado puede favorecer también la descomposición (7).

Como se ha indicado anteriormente, la sinterización se llevó a cabo en crisoles sellados con polvo de $\mathrm{ZrO}_{2}$ pero sin atmósfera rica en $\mathrm{PbO}$. Esto produjo una densificación a menor temperatura $\left(7.73 \pm 0.12 \mathrm{~g} / \mathrm{cm}^{3}\right.$ a $\left.1100^{\circ} \mathrm{C}\right)$ que en el caso del mismo PZT sinterizado en presencia de buffer $(7.75 \pm 0.19$ $\mathrm{g} / \mathrm{cm}^{3}$ a $1250^{\circ} \mathrm{C}$ ) (7) lo que indica que la mayor actividad del $\mathrm{PbO}$ en el material sinterizado sin buffer acelera su densificación. Este hecho puede tener importantes implicaciones industriales, no sólo debido al ahorro energético que conlleva la disminución de la temperatura de sinterización sino también debido a que el montaje del sistema de crisoles necesario durante la sinterización se simplifica.

\subsection{Microestructura}

El análisis de la microestructura mediante MEB revela que la evolución microestructural de los materiales estudiados es diferente. En las micrografías de ambos materiales sinterizados a $1100^{\circ} \mathrm{C}$ (Figura 2) se observa que en el A1 la distribución de tamaño de grano es bimodal $\left(\mathrm{D}_{\mathrm{g}}=3.7 \pm 0.2 \mu \mathrm{m}\right)$, al igual que ocurre en el A2. Sin embargo, en este último el tamaño de grano es menor $\left(\mathrm{D}_{\mathrm{g}}=2.0 \pm 0.2 \mu \mathrm{m}\right)$ y se aprecia un mayor número de poros. No sólo hay que tener en cuenta que esta mayor porosidad facilita la reacción de descomposición del PZT, lo que produce la aparición de $\mathrm{ZrO}_{2}$ (6). La reducción del tamaño de grano en el material A2 se produce por la presencia de una mayor cantidad de $\mathrm{Nb}_{2} \mathrm{O}_{5}$ ya que los iones del dopante se concentran cerca de los bordes de grano y reducen grandemente su movilidad (11).

La mayor presencia de $\mathrm{ZrO}_{2}$ en el material A2 se estudió cualitativamente mediante la relación de las áreas de los picos de difracción de Rayos X principales de la perovskita (1 10 ) y de la circona monoclínica (-1 11 1) en materiales sinterizados a $1100^{\circ} \mathrm{C}$ (Figura 3). Como puede verse, la presencia de $\mathrm{ZrO}_{2}$ es mucho menor en el material $\mathrm{A} 1\left(0.6 \% \mathrm{ZrO}_{2}\right)$ que en el $\mathrm{A} 2$ $\left(3.0 \% \mathrm{ZrO}_{2}\right)$. En los difractogramas de Rayos $\mathrm{X}$ también se ve claramente como esta descomposición produce el movimiento de la composición hacia la zona tetragonal del diagrama de fases $\mathrm{PbZrO}_{3}-\mathrm{PbTiO}_{3}$.

\subsection{Propiedades dieléctricas y piezoeléctricas}

El aumento de la temperatura de sinterización hace disminuir las propiedades eléctricas en ambos materiales debido a que las pérdidas de $\mathrm{PbO}$ producen un desplazamiento de la composición del PZT hacia la zona rica en $\mathrm{PbTiO}_{3}$ donde las propiedades eléctricas del PZT son menores (12).

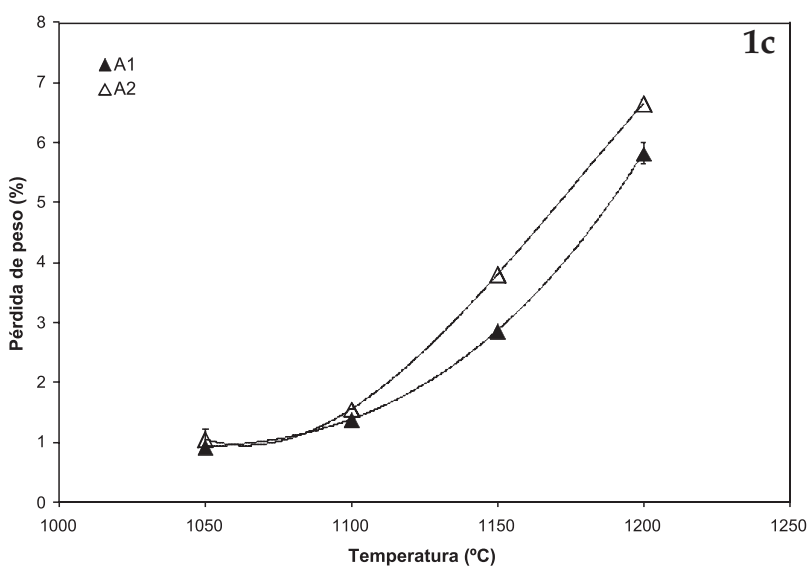

Figura 1. c) Pérdidas de peso en función de la temperatura de sinterización de los materiales A1 y A2.
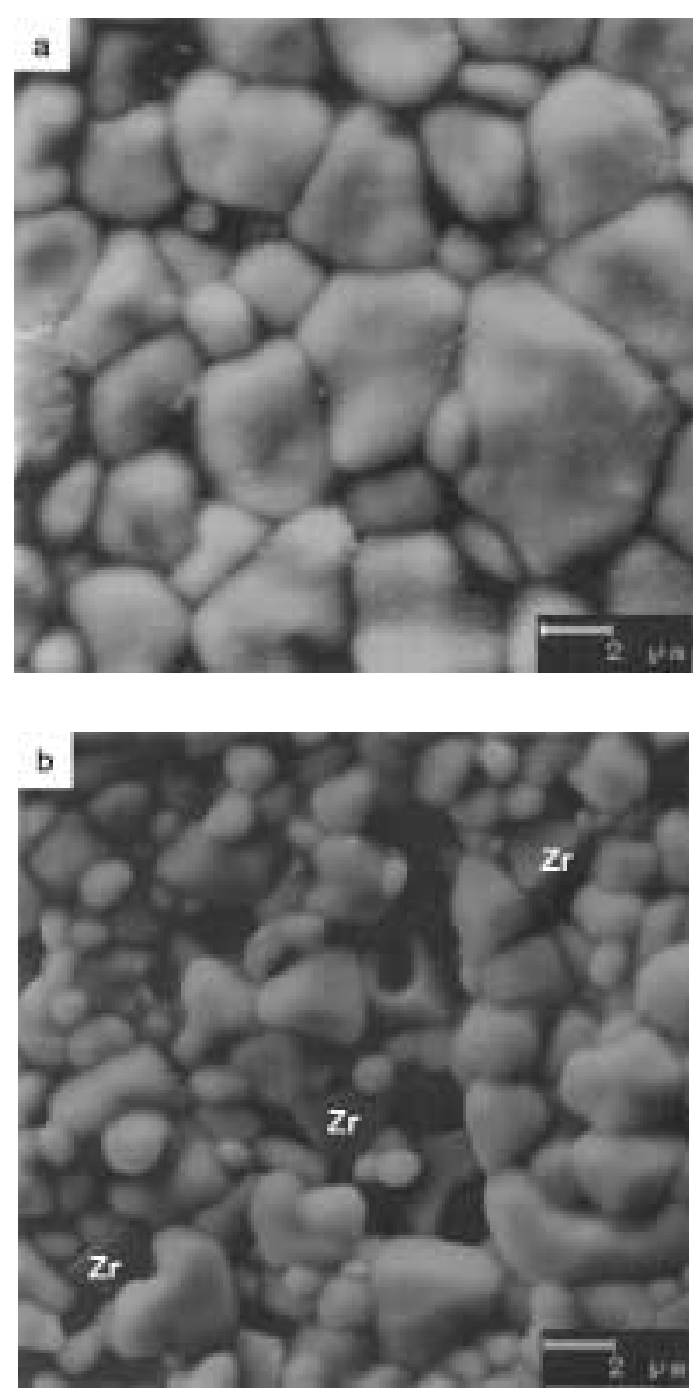

Figura 2. Micrografías de MEB de la superficie sinterizada sin modificar. a) Material A1 y b) material A2 ambos sinterizados a $1100^{\circ} \mathrm{C} / 4 \mathrm{~h}$. 


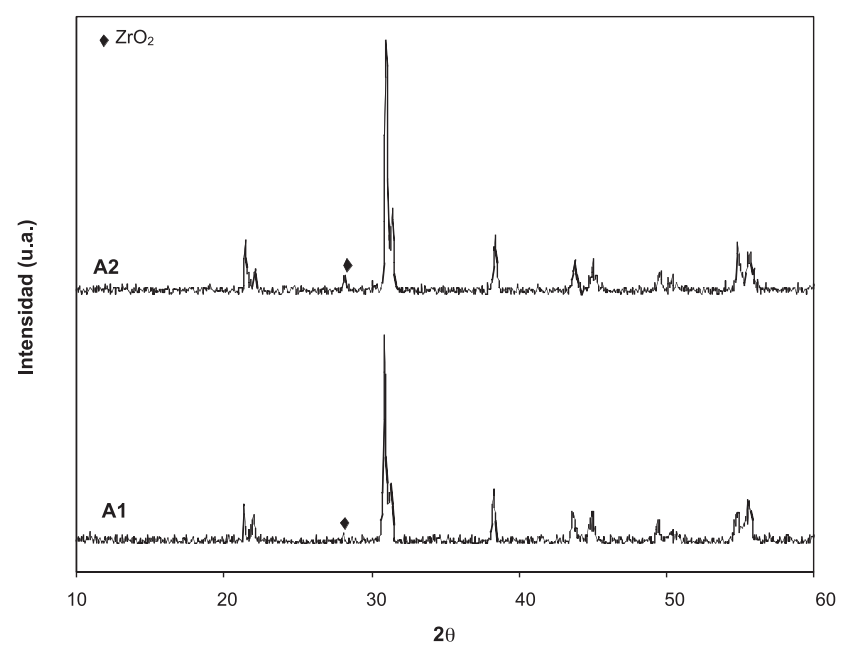

Figura 3. Difractogramas de Rayos X correspondientes a la superficie de los materiales A1 y A2 sinterizados a $1100^{\circ} \mathrm{C}$.

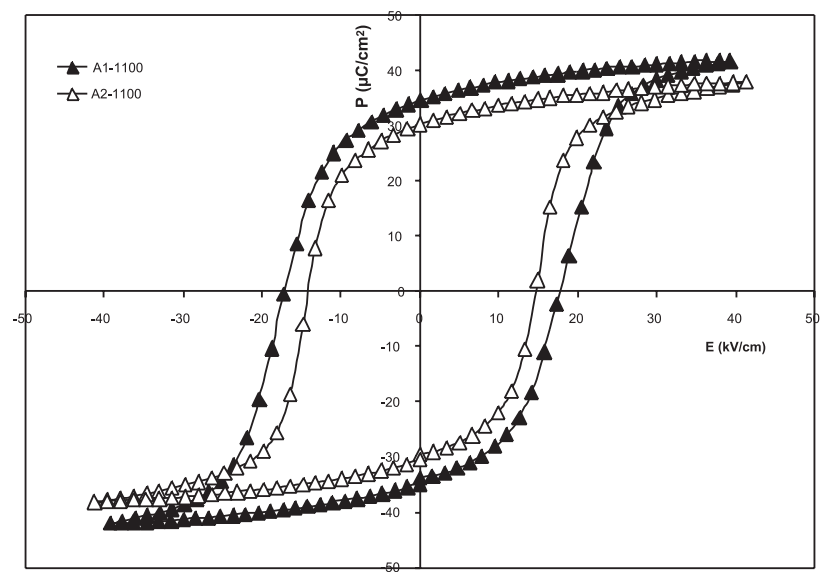

Figura 4. Curvas de histéresis correspondientes a los materiales A1 y A2 sinterizados a $1100^{\circ} \mathrm{C} / 4 \mathrm{~h}$.

En los ciclos de histéresis de ambos materiales sinterizados a $1100^{\circ} \mathrm{C}$ (Fig. 4) se observa que la polarización remanente es menor para el material A2. Esto se debe al menor tamaño de grano que presenta este material. Esta menor polarización junto con la menor densidad que presenta el material modificado hace que tanto $\mathrm{k}_{\mathrm{p}}$ como $\mathrm{d}_{33}$ también sean menores (Fig 5.a y 5.b). Además, la presencia de fases secundarias no ferroeléctricas provenientes de la descomposición del PZT ( $\mathrm{PbO}$ libre y $\mathrm{ZrO}_{2}$ ) provoca también la disminución de las propiedades piezoeléctricas en el material modificado.

Para estudiar el efecto del desplazamiento de la composición y la presencia de fases secundarias sobre las propiedades se estableció una correlación entre la temperatura de Curie experimental y la temperatura de Curie calculada a partir de la composición teórica del material teniendo en cuenta las pérdidas de $\mathrm{PbO}$ durante la sinterización. Para dicha correlación se tuvieron en cuenta las siguientes reacciones que pueden tener lugar en los materiales durante su sinterización:
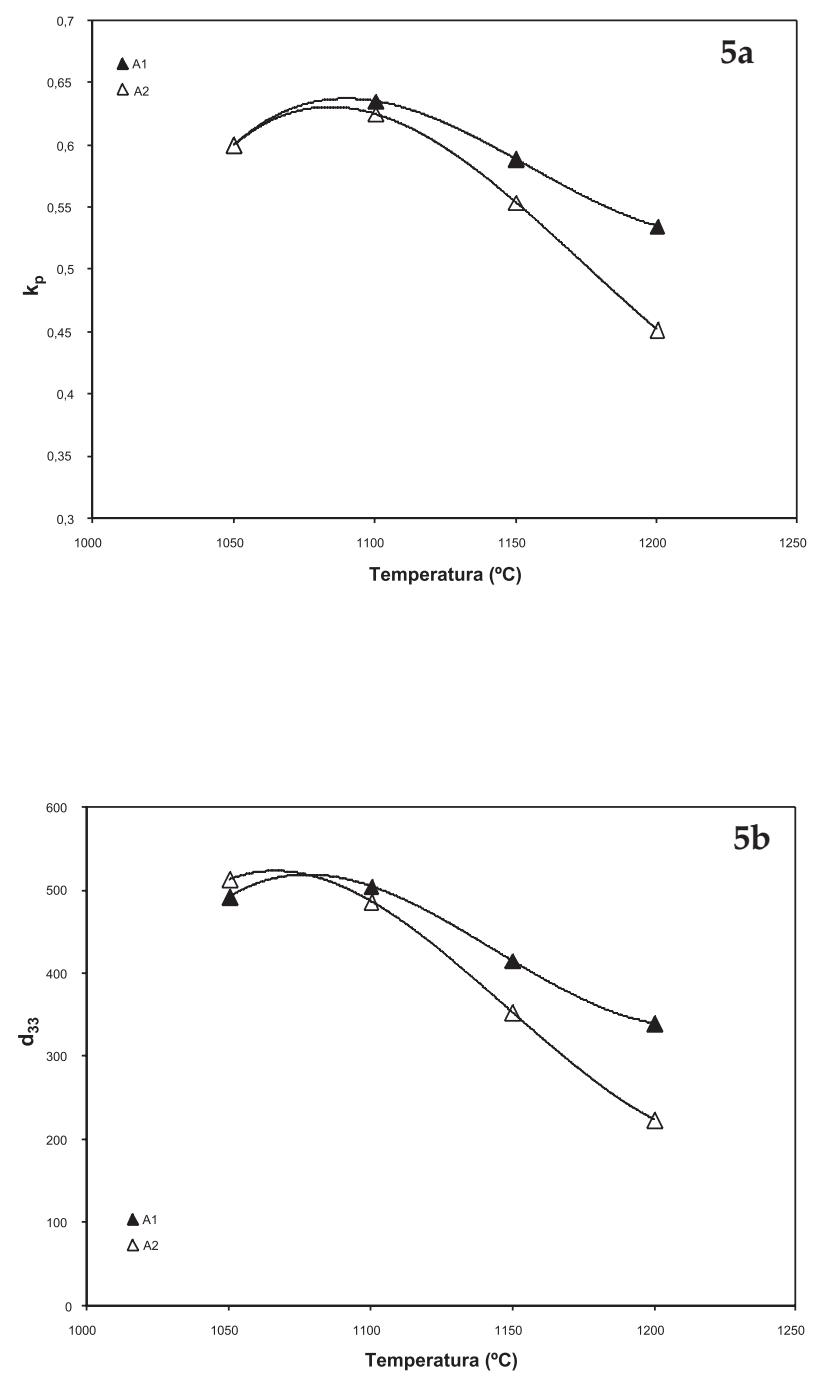

Figura 5. a) Variación de $k_{p}$ de los materiales A1 y A2 en función de la temperatura de sinterización. b) Variación de $\mathrm{d}_{33}$ de los materiales A1 y A2 en función de la temperatura de sinterización.

I. Ecuación de descomposición del PZT (6)

$$
\mathrm{Pb}\left(\mathrm{Zr}_{x} \mathrm{Ti}_{1-x}\right) \mathrm{O}_{3} \rightarrow \mathrm{Pb}_{(1--)}\left(\mathrm{Zr}_{x-\eta} \mathrm{Ti}_{1-x}\right) \mathrm{O}_{3}-\Delta-2 \eta+\Delta \mathrm{PbO}+\eta \mathrm{ZrO}_{2}
$$

II. Generación de vacantes por incorporación de $\mathrm{Nb}_{2} \mathrm{O}_{5}$ a la red (3)

$\mathrm{Pb}(\mathrm{Zr}, \mathrm{Ti}) \mathrm{O}_{3}+\mathrm{y} / 2 \mathrm{Nb}_{2} \mathrm{O}_{5} \rightarrow \mathrm{Pb}\left(\mathrm{Zr}, \mathrm{Ti}_{1} \mathrm{Nb}_{\mathrm{y}}\right) \mathrm{O}_{3}+\left(\mathrm{V}_{\mathrm{Pb}}\right)_{\mathrm{y} / 2}+\mathrm{y} / 2 \mathrm{PbO}$

Como puede verse en la Figura 6 en la que se representan los valores del Ec de los materiales A1 y A2 sinterizados a diferentes temperaturas, el campo coercitivo de los materiales A1 sinterizados a temperaturas mayores de $1100^{\circ} \mathrm{C}$ $(\mathrm{Ec}=18 \pm 0.5 \mathrm{kV} / \mathrm{cm})$ es menor que el Ec de las muestras sinterizadas a $1050^{\circ} \mathrm{C}(\mathrm{Ec}=22 \pm 0.5 \mathrm{kV} / \mathrm{cm})$. Esto indica que a partir de $1100^{\circ} \mathrm{C}$ se produce la incorporación del $\mathrm{Nb}_{2} \mathrm{O}_{5}$ a la red del PZT produciendo la disminución del campo coercitivo como corresponde a un dopante donador. Por el contrario, en el material A2 el campo coercitivo aumenta a partir de $1150^{\circ} \mathrm{C}$. 


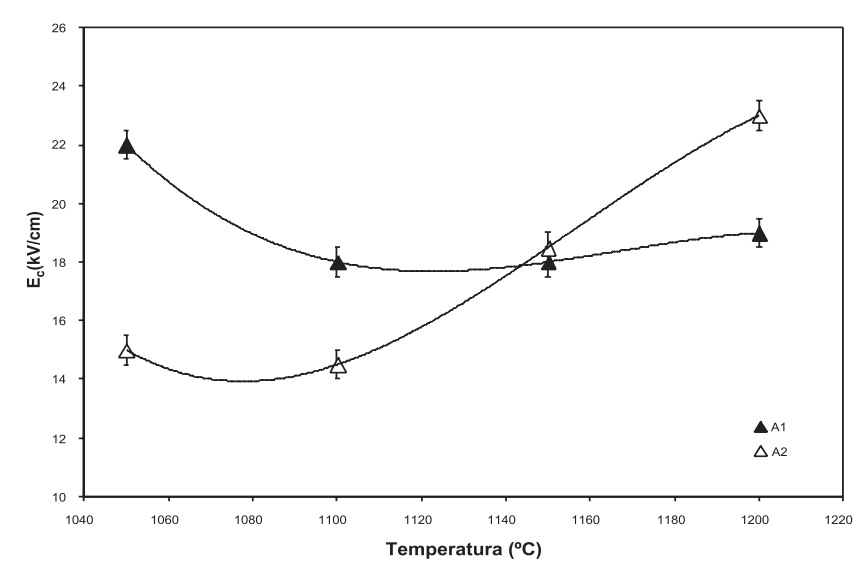

Figura 6. Variación de Ec en función de la temperatura de sinterización de los materiales A1 y A2.

Además, a baja temperatura $\left(1050^{\circ}-1100^{\circ} \mathrm{C}\right)$, el campo coercitivo de A2 es menor que en A1 (ver Figura 6), indicando que se ha incorporado una mayor cantidad de $\mathrm{Nb}_{2} \mathrm{O}_{5}$ a la red del PZT (el material A2 se modificó con un exceso de $\mathrm{Nb}_{2} \mathrm{O}_{5}$ ). Sin embargo, a $1150^{\circ} \mathrm{C}$ el Ec de los materiales A1 y A2 es muy similar $(\mathrm{Ec}=18 \pm 0.5 \mathrm{kV} / \mathrm{cm})$ por lo que en ambos casos la cantidad de $\mathrm{Nb}_{2} \mathrm{O}_{5}$ incorporado a la red del PZT es similar y sería la correspondiente a la formulación nominal del PZT-5A, es decir, a $1150^{\circ} \mathrm{C}$ el exceso de $\mathrm{Nb}_{2} \mathrm{O}_{5}$ adicionado en el material A2 no se incorpora al PZT. A $1200^{\circ} \mathrm{C}$ el Ec del material A2 $(E c=23 \pm 0.5 \mathrm{kV} / \mathrm{cm})$ es mayor que en el material $\mathrm{A} 1$ $(\mathrm{Ec}=18 \pm 0.5 \mathrm{kV} / \mathrm{cm})$ indicando que a esta temperatura el $\mathrm{Nb}_{2} \mathrm{O}_{5}$ (correspondiente al dopado y al exceso) no está incorporado a la red del PZT, lo que podría deberse a una extracción del $\mathrm{Nb}_{2} \mathrm{O}_{5}$ inducida por la gran cantidad de $\mathrm{PbO}$ libre presente a esta temperatura.

La curva experimental de variación de la temperatura de Curie en función de la temperatura de sinterización para A1 se ajusta bien a los datos (Figura 7, Tabla II) calculados teniendo en cuenta la incorporación completa del $\mathrm{Nb}_{2} \mathrm{O}_{5}$ correspondiente a la composición nominal del PZT-5A a partir de $1100^{\circ} \mathrm{C}$ y considerando que a $1050^{\circ} \mathrm{C}$ esta incorporación no se ha producido. Como se ha indicado antes, la incorporación de $\mathrm{Nb}^{5+}$ a la red del $\mathrm{PZT}$ genera vacantes en posición $\mathrm{A}$ (vacantes de $\mathrm{Pb}^{+2}$ ) y, por tanto, las pérdidas de $\mathrm{PbO}$ experimentales engloban el $\mathrm{PbO}$ que se pierde como consecuencia de la generación de estas vacantes [2] y el $\mathrm{PbO}$ que se pierde por volatilización y que es el que realmente produce la modificación de la composición del PZT y su alejamiento de la frontera morfotrópica de fase [1]. Estos hechos explican el aumento de la $\mathrm{T}_{\mathrm{c}}$ debido al desplazamiento de la composición hacia la zona rica en $\mathrm{PbTiO}_{3}$ y la disminución de las propiedades dieléctricas y piezoeléctricas a temperaturas mayores de $1100^{\circ} \mathrm{C}$.

En el caso del material con exceso de $\mathrm{Nb}_{2} \mathrm{O}_{5}$ el ajuste de la curva experimental con los datos calculados teniendo en cuenta los valores de Ec no es tan bueno como en el caso anterior, especialmente a alta temperatura. La razón más probable es que en el material permanezca PbO libre en forma de fase líquida que no puede considerarse en los cálculos ya que no contribuye a las pérdidas de peso. De hecho, en la Figura 8, correspondiente a la micrografía de MEB de la superficie del material A2 sinterizado a $1200^{\circ} \mathrm{C}$, puede verse gran cantidad de fase líquida rica en $\mathrm{PbO}$ que no ha llegado a volatilizarse.
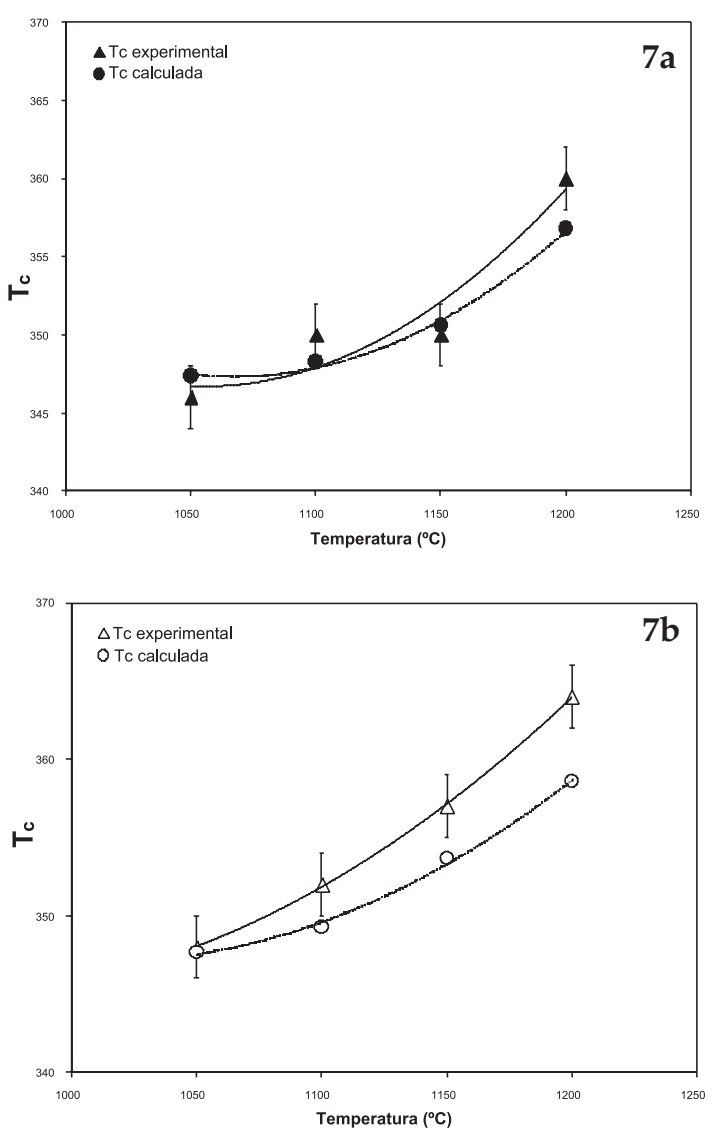

Figura 7. a) Correlación entre las curvas experimental y calculada de las temperaturas de Curie en función de la temperatura de sinterización para el material A1. b) Correlación entre las curvas experimental y calculada de las temperaturas de Curie en función de la temperatura de sinterización para el material A2.

TABLA II. TEMPERATURAS DE CURIE EXPERIMENTALES Y CALCULADAS EN FUNCIÓN DE LA INCORPORACIÓN DE $\mathrm{Nb}_{2} \mathrm{O}_{5}$ Y LAS PÉRDIDAS DE PESO EXPERIMENTALES PARA LOS MATERIALES A1 Y A2

\begin{tabular}{|c|c|c|c|c|}
\cline { 2 - 5 } \multicolumn{1}{c|}{} & \multicolumn{2}{c|}{$\mathrm{A} 1$} & \multicolumn{2}{c|}{$\mathrm{A}$} \\
\hline $\begin{array}{c}\text { Temperatura de } \\
\text { sinterización }\left({ }^{\circ} \mathrm{C}\right)\end{array}$ & $\begin{array}{c}\text { Tc experimental } \\
\left({ }^{\circ} \mathrm{C} \pm 2\right)\end{array}$ & Tc calculada & $\begin{array}{c}\text { Tc experimental } \\
\left({ }^{\circ} \mathrm{C} \pm 2\right)\end{array}$ & Tc calculada \\
\hline 1050 & 346 & 347.4 & 348 & 347.7 \\
\hline 1100 & 350 & 348.3 & 352 & 349.3 \\
\hline 1150 & 350 & 350.6 & 357 & 353.7 \\
\hline 1200 & 360 & 356.8 & 364 & 358.6 \\
\hline
\end{tabular}

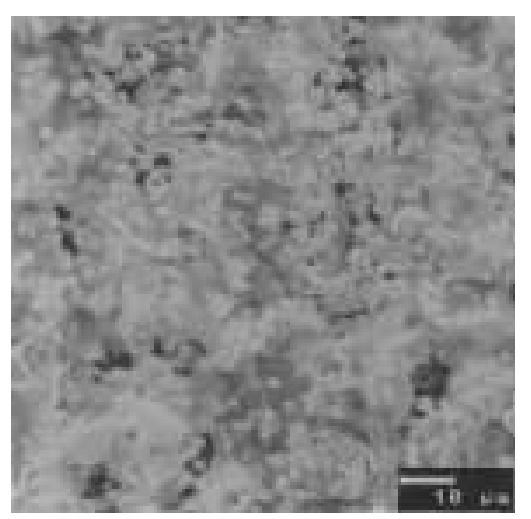

Figura 8. Micrografía de MEB correspondiente a la superficie sin modificar del material A2 sinterizado a $1200^{\circ} \mathrm{C} / 4 \mathrm{~h}$. 


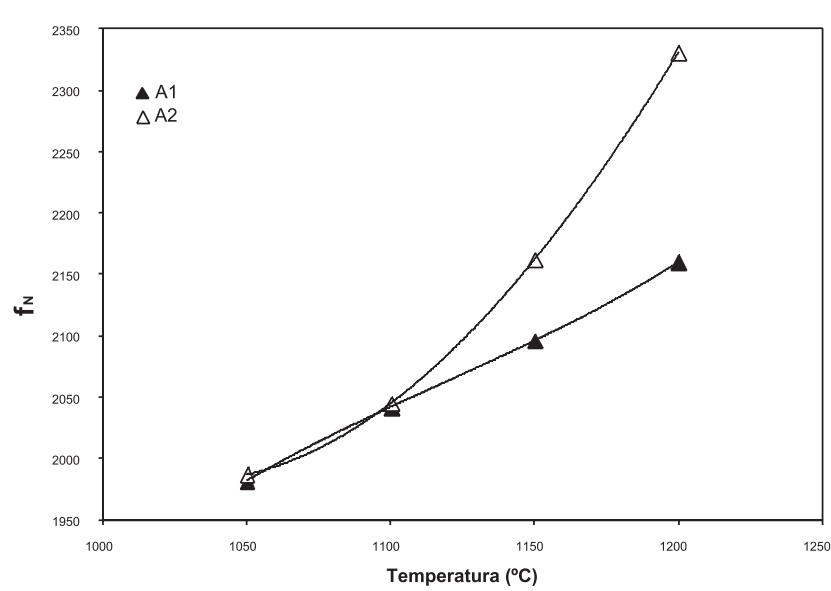

Figura 9. Variación de $\mathrm{f}_{\mathrm{N}}$ en función de la temperatura de sinterización para los materiales A1 y A2.

Esta fase líquida, que como se ha indicado no puede cuantificarse en las pérdidas de peso, sin embargo, ya no forma parte de la matriz de PZT cuya composición estaría entonces más desplazada hacia la zona rica en $\mathrm{PbTiO}_{3}$ y, por tanto, con una $\mathrm{T}_{\mathrm{c}}$ mayor, como indican los datos experimentales.

En la Figura 9 se representa la variaciación de la frecuencia característica $\left(\mathrm{f}_{\mathrm{N}}\right)$ con la temperatura de sinterización de los materiales A1 y A2. Como puede verse la $f_{N}$ aumenta al aumentar la temperatura de sinterización en ambos materiales, lo cual puede estar corroborando la presencia de una lámina inactiva de fase líquida que dificulta la vibración del PZT debido a un aumento de la rigidez mecánica del material o debido a que dicha fase inactiva puede estar actuando como un condensador en serie, formado por $\mathrm{ZrO}_{2}$ y la fase líquida rica en $\mathrm{PbO}$, con los granos de $\mathrm{PZT}$ por lo que sería necesaria más energía para conseguir la vibración de la matriz y, por tanto, se produciría el aumento de la $\mathrm{f}_{\mathrm{N}}$. El aumento de la $\mathrm{f}_{\mathrm{N}}$ en el material A2 es más acusado que en el A1 como corresponde a la mayor descomposición producida en el material modificado con exceso de $\mathrm{Nb}_{2} \mathrm{O}_{5}$.

\section{CONCLUSIONES}

La adición de un exceso de $\mathrm{Nb}_{2} \mathrm{O}_{5}$ y el procesamiento que implica, a un material de PZT-5A dopado con $\mathrm{Nb}_{2} \mathrm{O}_{5}$, conduce a una rápida descomposición del material. Cuando se estudia el material sin exceso de $\mathrm{Nb}_{2} \mathrm{O}_{5}$ la disminución de las propiedades eléctricas del material puede atribuirse al movimiento de la composición hacia la zona rica en $\mathrm{PbTiO}_{3}$ y a la disminución de la densidad del material al aumentar la tem- peratura. Sin embargo, debido a que este efecto es mucho más pronunciado en el material con exceso de $\mathrm{Nb}_{2} \mathrm{O}_{5}$ deben existir razones adicionales que justifiquen la drástica disminución de las propiedades como son la formación de fases secundarias por la aceleración en la descomposición del material, la existencia de fase líquida rica en $\mathrm{PbO}$ y la no incorporación del $\mathrm{Nb}_{2} \mathrm{O}_{5}$ en la red del PZT a altas temperaturas de sinterización, lo que convierte a éste en un material sin los efectos típicos de un dopante donador $y$, por tanto, con menores constantes dieléctricas y piezoeléctricas.

\section{AGRADECIMIENTOS}

Los autores quieren agradecer al CSIC, a la empresa KERABEN S.A. y al Proyecto EUREKA 2309 FACTORYPAMIS su apoyo en la realización de este trabajo.

\section{BIBLIOGRAFÍA}

1. G. H. Haertling. "Ferroelectric Ceramics: History and Techology". J. Am. Ceram. Soc. 82(4)797-818 (1999).

2. J. F. Fernández, P. Durán, C. Moure. “Materiales Cerámicos Ferroeléctricos y sus Aplicaciones". Bol. Soc. Esp. Ceram. Vidr., 32 (1) 5-15 (1993).

3. S. Takahashi. "Effects of Impurity Doping in Lead Zirconate-Titanate Ceramics". Ferroelectrics 41, 143-156 (1982).

4. T. Ikeda, Y. Tanaka, T. Ayakawa, H. Noake. "Precipitation of Zirconia Phase in Niobium-Modified Ceramics of Lead Zirconate Titanate". Jpn. J. Appl. Phys., 3(10), 581-587 (1964).

5. A.E. Park, B. Kim, K. Song, S. Park. "Piezoelectrics Properties of $\mathrm{Nb}_{2} \mathrm{O}_{5}$ Doped and $\mathrm{MnO}_{2}-\mathrm{Nb}_{2} \mathrm{O}_{5}$ co-doped $\mathrm{Pb}\left(\mathrm{Zr}_{0.53} \mathrm{Ti}_{0.47}\right) \mathrm{O}_{3}$ Ceramics". J. Mat. Sci., 6, 97-101 (1997).

6. A.C. Caballero, E. Nieto, P. Durán, C. Moure, M. Kosec, Z. Samardzija, G. Drazic. "Ceramic-Electrode Interaction in PZT and PNN-PZT Multilayer Ceramics with Ag70/Pd30 Inner Electrode". J. Mat. Sci., 32, 3257-3262 (1997).

7. L. A. Celi, A. C. Caballero, M. Villegas, P. Durán, C. Moure, J. F. Fernández. "Efecto de las Características de los Polvos Cerámicos sobre la Densificación de Materiales Cerámicos PZT". Bol. Soc. Esp. Ceram. Vidr., 38 (5) 493-497 (1999).

8. L. L. Fullman, "Measurement of Particle Size in Opaque Bodies". Trans Aime, 197, 447 (1953).

9. Piezoelectric IEEE Standards on Piezoelectricity, ANSI/IEEE Sttd. 176. The Institute of Electrical and Electronics Engineers, New York, USA (1987).

10. M. Villegas, J.F. Fernández, A.C. Caballero, Z. Samardija, G. Drazic, M. Kosec. "Effects of PbO Excess in $\mathrm{Pb}\left(\mathrm{Mg}_{1 / 3} \mathrm{Nb}_{2 / 3}\right) \mathrm{O}_{3}-\mathrm{PbTiO}_{3}$ Ceramics: Part II: Microstructure Development". J. Mat. Res. 14, 898-905 (1999).

11. R.B. Atkin, R.M. Fulrath. "Point Defects and Sintering of Lead Zirconate Titanate". J. Am. Ceram. Soc., 54(5), 265-270 (1971).

12. B. Jaffe, R.S. Roth, S. Marzullo. "Piezoelectric Properties of Lead Zirconate Titanate Solid Solution Ceramics". J. Appl. Phys., 25, 809-810 (1954).

Recibido: 31.05 .01

Aceptado: 10.12 .01 